\title{
Production of Valuable Heavy Hydrocarbon Fuel Oil by Thermal Degradation Process of Post-Consumer Municipal Polystyrene (PS) Waste Plastic in Steel Reactor
}

\author{
Moinuddin Sarker ${ }^{*}$, Mohammad Mamunor Rashid, Muhammad Sadikur Rahman, Mohammed Molla
}

Natural State Research, Inc. Department of Research, Development, 37 Brown House Road (2 ${ }^{\text {nd }}$ Floor), Stamford, CT 06902, USA

\begin{abstract}
Heavy hydrocarbon fuel oil production was conducted only Polystyrene (PS) waste plastic and temperature range was used for this experiment $200-450{ }^{\circ} \mathrm{C}$ and thermal degradation process utilized. Fractional colu mn temperature range was used for heavy fuel oil collection $340-365^{\circ} \mathrm{C}$. Polystyrene waste plastic was used only this experiment and experiment was performed without catalyst in the batch process under laboratory fume hood. Produced fuel was analysis by Gas Chromatography and Mass Spectrometer (GC/MS) and FT-IR. GC/MS analysis result indicates that produced fuel hydrocarbon chian range $\mathrm{C}_{6}$ to $\mathrm{C}_{25}$ and FT-IR analysis result provided produced fuel functional group band energy which is reflect with calorific value. It was stated that proper selection of the process parameter and make it possible to control and limited grade fuels production product distribution such as valuable hydrocarbon fuel oil and other grade fuels fraction. Basic analys is of heavy hydrocarbon fueloil GC/MS and FT-IR results described in the result and discussion section. Produced fuel sulphur was determined by ASTM test method and sulphur content was less then environmental protection agency (EPA) level. Fuel could be used for feed stock refinery or heavy equipment because produce fuel has hydrocarbon range is $\mathrm{C}_{6}$ to $\mathrm{C}_{25}$.
\end{abstract}

Keywords Hydrocarbon Fuel Oil, Thermal Degradation, Polystyrene, Waste Plastic, PS, Heavy Fue1

\section{Introduction}

Plastic materials are present in almost every area of daily life. However, the significant growth of plastic consumption also led to increasing a mounts of waste plastics. The disposal of plastic wastes is an important environmental problem all over the world. Europe is generating about 15 million tons of post consumer plastic waste[1] while the United States alone generates more than 20 million tons of plastic wastes each year[2].

Recently, the degradation of polymers into liquid hydrocarbons has attracted much attention from the viewpoint of the utilization of waste plastics as an energy resource. There have been many reports on the conversion of plastics to fuels using solid acid catalysts; especially on polyethylene (PE) and polypropylene (PP)[3-8] Solid acid catalysts generally have been preferred for polymer degradation because of their high cracking abilities [9-10].

Nowadays, plastics are one of the most used materials due to their characteristics.

The world production of plastics rose to a value of $169 \mathrm{MT}$ in 2003, while materials such as aluminium, very much used

* Corresponding author:

msarker@naturalstat eres earch.com (Moinuddin Sarker)

Published online at http://journal.sapub.org/ep

Copyright (C) 2012 Scientific \& Academic Publishing. All Rights Reserved in many applications for years, only showed a world production of $28 \mathrm{MT}$ in the same year[11]. This fact has caused the development of different techniques for the elimination of plastics, taking advantage of this type of material. Basically, four methods for the elimination of plastics can be distinguished: mechanical recycling, landfilling, incineration, and chemical recycling,[12-14] although they present several disadvantages. In one way, mechanical recycling (or secondary recycling) can only be applied to thermoplastic materials;[13] in the case of landfilling, the space is limited and most of the plastic wastes are resistant to environmental degradation; $[13,14]$ incineration (or quaternary recycling) is an interesting alternative because of the energy production but less attractive from an environmental point of view[12,14]. Finally, chemical recyc ling (or tertiary recycling) imp lies the conversion of polymers into more valuable chemicals or fuels[12-13].

In this type of recycling method, pyrolysis is included as an interesting alternative for the elimination of plastics. The hydro-conversion process of heavy oil and residue is one of the main processes for converting a heavy carbonaceous feedstock to lower-boiling products. Generally heterogeneo us catalysts, such as sulfide of cobalt, molybdenum, or nickel supported by alumina or silica-alumina, are used in the process. The constituents having higher molecular weight in heavy oil and residue deposit on the surface of the catalyst, 
block the pores of the catalyst, and then result in rapid decline of the hydrogenation activity[15]. Slurry-phase hydro-cracking of heavy oil was first used in Germany as early as 1929 for hydrogenation of coal, and two units were successfully working during World War II, which were switched to vacuum residue (VR) feed and operated until 1964[16]. In today's world, research on slurry-phase hydro-cracking processes is very active. There are now more than 10 such technologies that is in pilot stage. Some of them have already had industrialized application.

\section{Experimental Process}

Polystyrene waste plastic collected from local restaurant and it was transparent food container and food particle was stuck with food container. PS waste plastic was washed with soap and cut into small pieces for liquefaction process. PS waste plastic cutting size was 2-3 inch and it was transfer into reactor chamber. Reactor inside sample was placed 500 gm. Reactor set up was properly under laboratory fume hood and it was make tighten properly every part (figure1). Distillation column was set up for fuel collection properly. Distillation colu mn temperature profile was setup fuel grade wise such as gasoline grade fuel collection temperature was 40-65 ${ }^{\circ} \mathrm{C}$, Naphtha Chemical grade temperature was $110-135^{\circ} \mathrm{C}$, Kerosene grade fuel collection temperature was $180-205^{\circ} \mathrm{C}$, diesel fuel grade temperature profile was $260-285^{\circ} \mathrm{C}$ and finally heavy fuel oil grade collection temperature was $340-365^{\circ} \mathrm{C}$. Reactor temperature profile was starting at $200^{\circ} \mathrm{C}$ to finished temperature $450^{\circ} \mathrm{C}$. After fin ished setup all te mperature profile and setup every part for PS waste plastic to fractional heavy fuel production then start electrical heating for liquefaction process. In this experiment was main goal heavy fueloil collection from PS waste plastic. When heated up PS waste plastic from $200^{\circ} \mathrm{C}$ to $450^{\circ} \mathrm{C}$ gradually at rate $15^{\circ} \mathrm{C} / 20 \mathrm{~min}$. we noticed that when temperature close to $320^{\circ} \mathrm{C}$ fuel vapor start to co me out. PS plastic melting point temperature is $260^{\circ} \mathrm{C}$ and it has aromatic group compound also. Temperature increasing gradually and PS waste plastic long chain hydrocarbon bond was break down and from as a short chain hydrocarbon compound. This experiment did not apply any extra chemical or catalyst only thermal degradation process was applied. Melted waste PS plastic was turn vapor when temperature was increased step by step and turn into liquid form at the end collected different grade fuel as temperature profile wise. Experimental process showed every step fuel collection, gas cleaning process and transfer light gas into Teflon bag. Whole process finished electricity input was 3.1 $\mathrm{kWh}$. From experimental process mass balance gasoline grade fuel collection $50 \mathrm{gm}(10 \%)$, naphtha chemical grade collection $100 \mathrm{gm}(20 \%)$, kerosene grade fuel collection 120 gm (24\%), diesel fuel collection $130 \mathrm{gm}(26 \%)$ and finally heavy fuel oil grade $40 \mathrm{gm}(8 \%)$. Produced heavy fuel oil density is $0.93 \mathrm{~g} / \mathrm{ml}$. From this experiment light gas was created $20 \mathrm{gm}(4 \%)$ sample and leftover residue was $40 \mathrm{gm}$ $(8 \%)$.

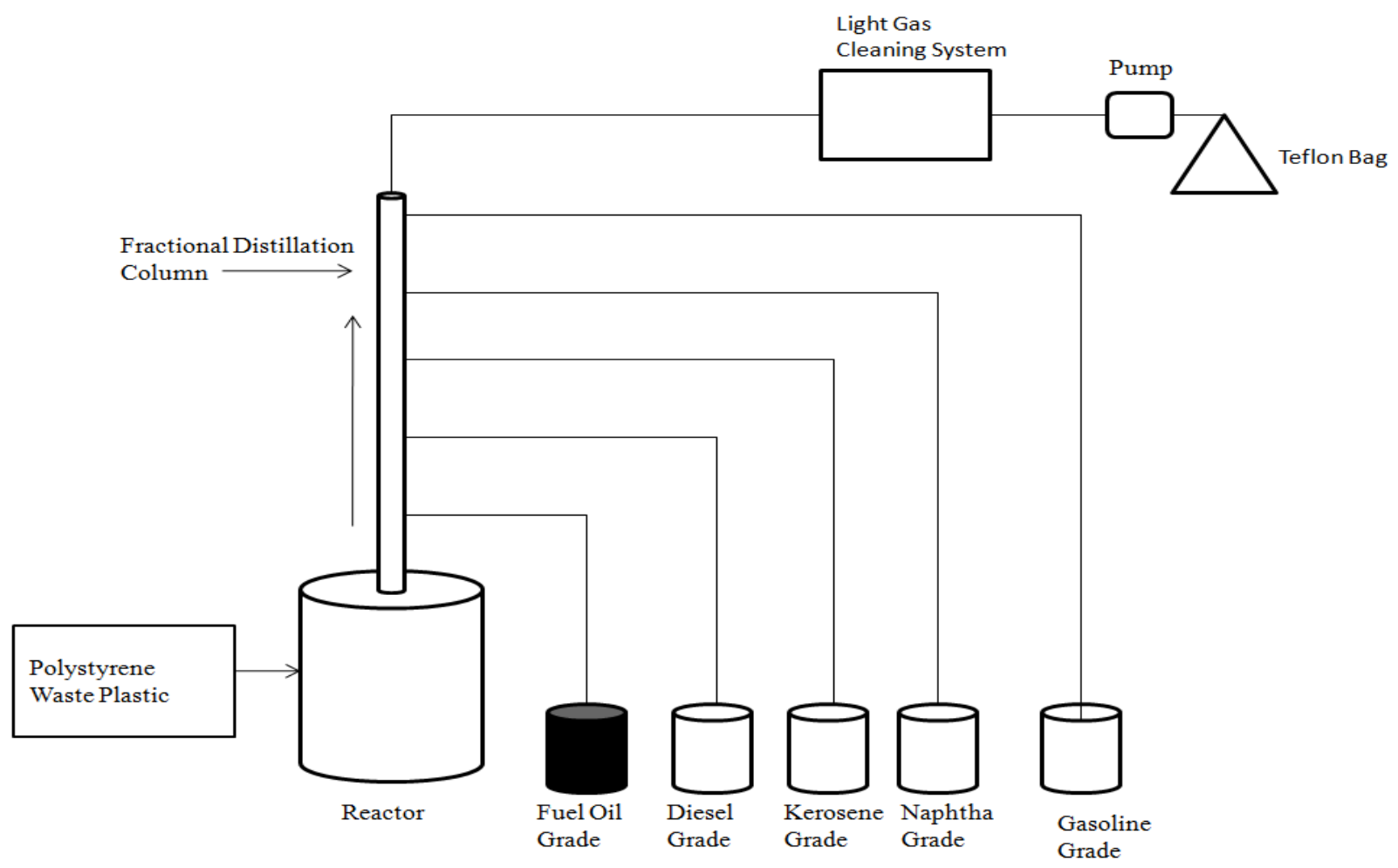

Figure 1. Polystyrene (PS) waste plast ic to heavy fuel production process 


\section{Result and Discussion}

From Perkin Elmer GC-MS (Model Clarus 500) analysis of PS waste plastic to heavy hydrocarbon fractional fuel (fig. $2 \&$ table 1) in accordance with the various retention times and trace masses different types of hydrocarbon compound and benzene derivatives compounds are appeared in the analysis result index. Many compounds are emerged on the analysis carbon range $\mathrm{C} 6$ to $\mathrm{C} 24$ among those few of them are elaborated in the analysis. Based on the retention time and trace mass following hydrocarbon compounds as follows such as at the initial phase of the analys is at retention time 3.28 and trace mass 78, compound is Benzene (C7H8), retention time 4.85 and trace mass 92 , compound is 1,5-Heptadiene-3-yne (C7H8), retention time 6.47 and trace mass 65 , compound is Ethyl benzene $(\mathrm{C} 8 \mathrm{H} 10)$, retention time 7.00 and trace mass 104 ,compound is Styrene, (C8H8), retention time 7.88 and trace mass 117 , compound is Benzene,2-propenyl- (C9H10), retention time 8.99 and trace mass 105, compound is Benzene, (1-methylpropyl)(C10H14), retention time 9.93 and trace mass 105,compound is Acetophenone $(\mathrm{C} 8 \mathrm{H} 8 \mathrm{O})$, retention time 10.87 and trace mass 117,compound name is Benzene, 1-butenyl-, (E)-, $(\mathrm{C} 10 \mathrm{H} 12)$, retention time 11.96 and trace mass 128 ,compound is Naphthalene $(\mathrm{C} 10 \mathrm{H} 8)$, retention time 12.91 and trace mass 91, compound is Benzene, hexyl$(\mathrm{C} 12 \mathrm{H} 18)$, retention time 13.59 and trace mass 142 , compound is Naphthalene, 2-methyl- $(\mathrm{C} 11 \mathrm{H} 10)$, retention time 14.93 and trace mass 168 , compound is 1,1'-Biphenyl, 3-methyl- (C13H12), retention time 15.39 and trace mass 167 , compound is Diphenylmethane, $(\mathrm{C} 13 \mathrm{H} 12)$, retention time 16.91 and trace mass 105 , compound is Benzene, 1,1'-(1-methyl-1,2-ethanediyl)bis- ( $\mathrm{C} 15 \mathrm{H} 16)$, retention time
17.79 and trace mass 119 , compound is Benzene, 2,6-dimethyl-1-(phenylmethyl)-, ( $\mathrm{C} 15 \mathrm{H} 16)$, retention time 18.88 and trace mass 91, compound is Benzene, 1,1'-(2-butene-1,4-diyl)b is-, ( C16H16), retention time 19.74 and trace mass 115, compound is Benzene, 1,1'-(1-butenylidene)bis- $(\mathrm{C} 16 \mathrm{H} 16)$. Also at retention time 20.69 and trace mass 105, compound is Benz[a]anthracene, 7,12-dihydro-- $(\mathrm{C} 18 \mathrm{H} 14)$, retention time 21.83 and trace mass 218, compound is 9-Phenyl-5H-benzocycloheptene, (C17H14) etc. In the final phase of the analysis index several compound are detected as according to their retention time and trace masses such as retention time 25.61 and trace mass 91, compound is Benzene, 1,1'-(1,3-propanediyl)b is(C15H16), retention time 27.82 and trace mass 306 , compound is $1,1^{\prime}: 2^{\prime}, 1^{\prime \prime}: 2^{\prime \prime}, 1^{\prime \prime}$-Quaterphenyl (C24H18), and ultimately retention time 29.70 and retention time 306 , compound is $1,1^{\prime}: 3^{\prime}, 1^{\prime \prime}$-Terphenyl, 5'-phenyl- (C24H18) as well.

Polystyrene (PP) waste plastic to heavy hydrocarbon fuel (fig.3) was analysis by Perkin Elmer DSC for fuel boiling point measuring and enthalpy value determination. PS heavy hydrocarbon fuel similar to fuel oil and this fuel heavy fuel collection temperature range was $340-365{ }^{\circ} \mathrm{C}$ by using fractional distillation column. Produced heavy hydrocarbon fractional fuel boiling point peak temperature is $140.43^{\circ} \mathrm{C}$ and peak height $82.4178 \mathrm{~mW}$. Fuel boil start temperature $\mathrm{X} 1=5.21^{\circ} \mathrm{C}, \mathrm{Y} 1=0.0129 \mathrm{~mW}$ and $\mathrm{X} 2=393.47^{\circ} \mathrm{C}, \mathrm{Y} 2=$ $1.5373 \mathrm{~mW}$. Onset temperature is $139.76^{\circ} \mathrm{C}$ and peak end temperature is $146.64^{\circ} \mathrm{C}$. Heat enthalpy delta $\mathrm{H}$ value is $14385.2266 \mathrm{~J} / \mathrm{g}$ and area is $14385.227 \mathrm{~mJ} .8 .84 \%$ fuel was boiling at $50^{\circ} \mathrm{C}$ and $100 \%$ was $392.11^{\circ} \mathrm{C}$.

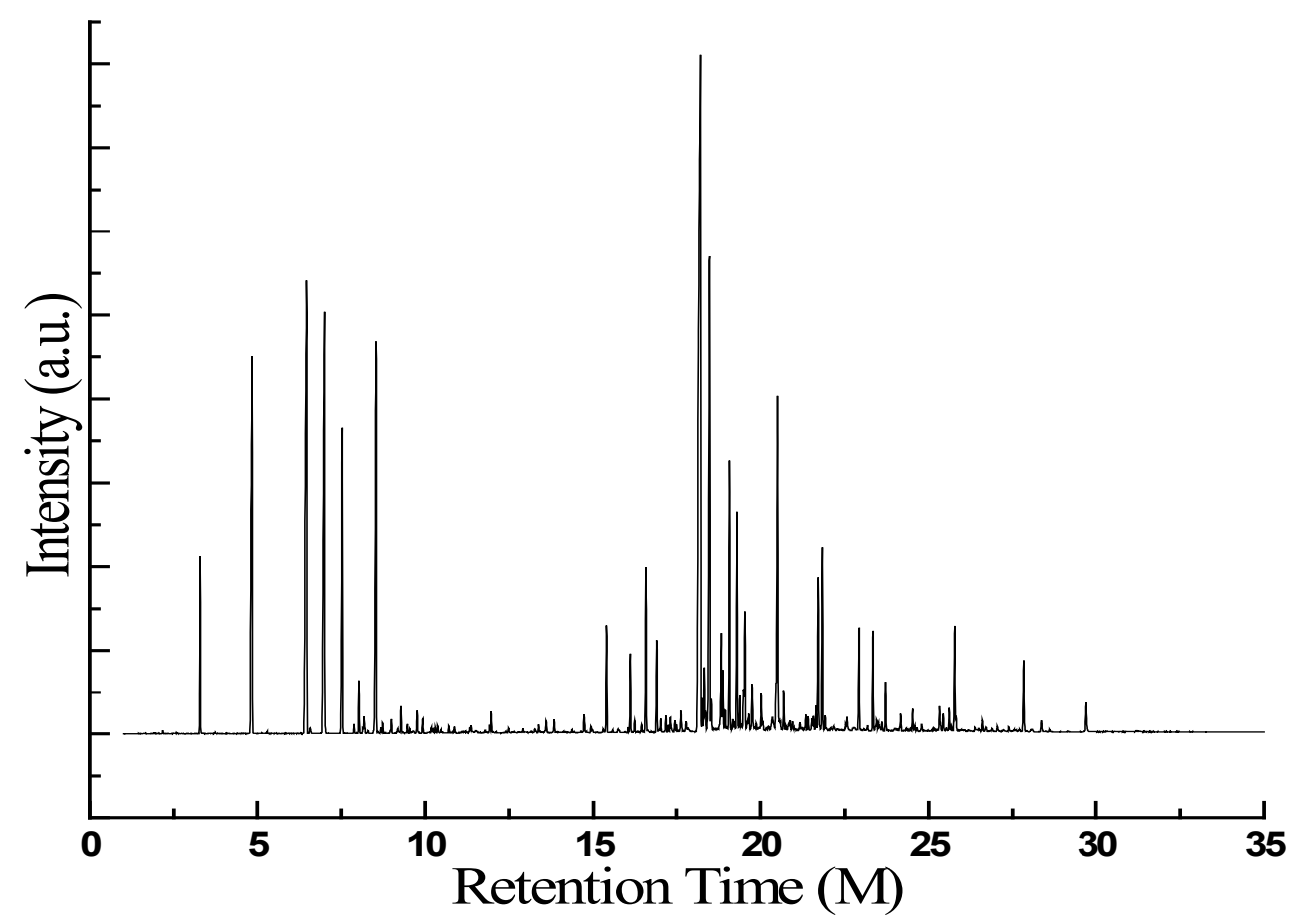

Figure 2. GC/MS Chromatogram of PS waste plast ic to heavy fuel oil 
Table 1. GC/MS Chromatogram compound lists of PS waste plastic to fuel

\begin{tabular}{|c|c|c|c|c|c|c|}
\hline $\begin{array}{l}\text { Number of } \\
\text { Peak }\end{array}$ & $\begin{array}{l}\text { Retention } \\
\text { Time }(\mathrm{M})\end{array}$ & $\begin{array}{c}\text { Trace } \\
\text { Mass }(\mathrm{m} / \mathrm{z})\end{array}$ & $\begin{array}{l}\text { Compound } \\
\text { Name }\end{array}$ & $\begin{array}{l}\text { Compound } \\
\text { Formula }\end{array}$ & $\begin{array}{l}\text { Molecular } \\
\text { Weight }\end{array}$ & Probability \% \\
\hline 1 & 3.28 & 78 & Benzene & C6H6 & 78 & 39.0 \\
\hline 2 & 4.85 & 92 & 1,5-Heptadien-3-yne & C7H8 & 92 & 31.7 \\
\hline 3 & 6.47 & 65 & Ethylbenzene & C8H1 0 & 106 & 20.4 \\
\hline 4 & 7.00 & 104 & Styrene & $\mathrm{C} 8 \mathrm{H} 8$ & 104 & 30.8 \\
\hline 5 & 7.53 & 105 & Benzene, 1-ethyl-2-methyl- & C9H12 & 120 & 18.9 \\
\hline 6 & 7.88 & 117 & Benzene, 2-propenyl- & С9H1 0 & 118 & 19.1 \\
\hline 7 & 8.03 & 91 & Benzene, propyl- & $\mathrm{C} 9 \mathrm{H} 12$ & 120 & 78.5 \\
\hline 8 & 8.19 & 77 & Benzaldehyde & $\mathrm{C} 7 \mathrm{H} 6 \mathrm{O}$ & 106 & 69.0 \\
\hline 9 & 8.54 & 103 & $\alpha$-Methylstyrene & С9H1 0 & 118 & 46.2 \\
\hline 10 & 8.73 & 57 & Decane & $\mathrm{C} 10 \mathrm{H} 22$ & 142 & 13.1 \\
\hline 11 & 8.99 & 105 & Benzene, (1-methylpropyl)- & $\mathrm{C} 10 \mathrm{H} 14$ & 134 & 28.9 \\
\hline 12 & 9.18 & 117 & Benzene, (2-methyl-1-propenyl)- & $\mathrm{C} 10 \mathrm{H} 12$ & 132 & 16.3 \\
\hline 13 & 9.28 & 117 & Benzene, 2-propenyl- & $\mathrm{C} 9 \mathrm{H} 10$ & 118 & 23.3 \\
\hline 14 & 9.47 & 117 & Indane & C9H10 & 118 & 20.2 \\
\hline 15 & 9.54 & 91 & Benzene, 3-butenyl- & $\mathrm{C} 10 \mathrm{H} 12$ & 132 & 60.1 \\
\hline 16 & 9.76 & 91 & Benzene, butyl- & $\mathrm{C} 10 \mathrm{H} 14$ & 134 & 27.1 \\
\hline 17 & 9.93 & 105 & Acetophenone & $\mathrm{C} 8 \mathrm{H} 8 \mathrm{O}$ & 120 & 31.7 \\
\hline 18 & 10.18 & 117 & Benzene, (2-methyl-1-propenyl)- & $\mathrm{C} 10 \mathrm{H} 12$ & 132 & 19.1 \\
\hline 19 & 10.37 & 43 & Undecane & $\mathrm{C} 11 \mathrm{H} 24$ & 156 & 41.2 \\
\hline 20 & 10.47 & 105 & Benzene, (1-methylbutyl)- & C1 $1 \mathrm{H} 16$ & 148 & 38.7 \\
\hline 21 & 10.70 & 117 & Benzene, 1-methyl-4-(2-propenyl)- & $\mathrm{C} 10 \mathrm{H} 12$ & 132 & 11.6 \\
\hline 22 & 10.87 & 117 & Benzene, 1-butenyl-, (E)- & $\mathrm{C} 10 \mathrm{H} 12$ & 132 & 17.4 \\
\hline 23 & 11.92 & 43 & Dodecane & $\mathrm{C} 12 \mathrm{H} 26$ & 170 & 11.5 \\
\hline 24 & 11.96 & 128 & Naphthalene & $\mathrm{C} 10 \mathrm{H} 8$ & 128 & 43.7 \\
\hline 25 & 12.91 & 91 & Benzene, hexyl- & $\mathrm{C} 12 \mathrm{H} 18$ & 162 & 60.0 \\
\hline 26 & 13.26 & 41 & Benzene, cyclohexyl- & $\mathrm{C} 12 \mathrm{H} 16$ & 160 & 9.65 \\
\hline 27 & 13.37 & 43 & Tridecane & $\mathrm{C} 13 \mathrm{H} 28$ & 184 & 11.7 \\
\hline 28 & 13.59 & 142 & Naphthalene, 2-methyl- & $\mathrm{C} 11 \mathrm{H} 10$ & 142 & 21.3 \\
\hline 29 & 14.36 & 91 & Benzene, heptyl- & $\mathrm{C} 13 \mathrm{H} 20$ & 176 & 49.2 \\
\hline 30 & 14.72 & 154 & Biphenyl & $\mathrm{C} 12 \mathrm{H} 10$ & 154 & 68.9 \\
\hline 31 & 14.93 & 168 & 1,1'-Biphenyl, 3-methyl- & $\mathrm{C} 13 \mathrm{H} 12$ & 168 & 23.6 \\
\hline 32 & 15.39 & 167 & Diphenylmethane & $\mathrm{C} 13 \mathrm{H} 12$ & 168 & 66.9 \\
\hline 33 & 16.03 & 43 & Tetradecane & $\mathrm{C} 14 \mathrm{H} 30$ & 198 & 10.7 \\
\hline 34 & 16.10 & 167 & Benzene, 1,1'-ethylidenebis- & $\mathrm{C} 14 \mathrm{H} 14$ & 182 & 33.3 \\
\hline 35 & 16.23 & 168 & 1,1'-Biphenyl, 4-methyl- & $\mathrm{C} 13 \mathrm{H} 12$ & 168 & 35.1 \\
\hline 36 & 16.56 & 91 & Benzene, $1,1^{\prime}-(1,2$-ethanediyl)bis- & $\mathrm{C} 14 \mathrm{H} 14$ & 182 & 92.9 \\
\hline 37 & 16.91 & 105 & $\begin{array}{c}\text { Benzene, } \\
\text { 1,1'-(1-methyl-1,2-ethanediyl)bis- }\end{array}$ & $\mathrm{C} 15 \mathrm{H} 16$ & 196 & 70.6 \\
\hline 38 & 17.03 & 179 & Phenanthrene, 9,10-dihydro-1-methyl- & $\mathrm{C} 15 \mathrm{H} 14$ & 194 & 52.8 \\
\hline 39 & 17.32 & 105 & 3-Phenylbicyclo[3.2.1]octa-2,6-diene & $\mathrm{C} 14 \mathrm{H} 14$ & 182 & 20.1 \\
\hline 40 & 17.45 & 167 & 1,1'-Biphenyl, 2-ethyl- & $\mathrm{C} 14 \mathrm{H} 14$ & 182 & 24.4 \\
\hline 41 & 17.50 & 103 & 1,2-Diphenylcyclopropane & $\mathrm{C} 15 \mathrm{H} 14$ & 194 & 55.9 \\
\hline 42 & 17.63 & 105 & $\begin{array}{c}\text { Benzene, } \\
\text { 1,1'-(1-methyl-1,2-ethanediyl)bis- }\end{array}$ & $\mathrm{C} 15 \mathrm{H} 16$ & 196 & 36.8 \\
\hline 43 & 17.79 & 119 & Benzene, 2,6-dimethyl-1-(phenylmethyl)- & $\mathrm{C} 15 \mathrm{H} 16$ & 196 & 19.3 \\
\hline 44 & 18.21 & 65 & $\begin{array}{c}\text { Benzene, } \\
\text { 1,1'-(1-methyl-1,2-ethanediyl)bis- }\end{array}$ & $\mathrm{C} 15 \mathrm{H} 16$ & 196 & 16.1 \\
\hline 45 & 18.48 & 105 & $\begin{array}{c}\text { Benzene, } \\
1,1^{\prime} \text {-(1-methyl-1,3-propanediyl)bis- }\end{array}$ & $\mathrm{C} 16 \mathrm{H} 18$ & 210 & 91.1 \\
\hline 46 & 18.84 & 179 & cis-Stilbene & $\mathrm{C} 14 \mathrm{H} 12$ & 180 & 28.9 \\
\hline 47 & 18.88 & 91 & Benzene, 1,1'-(2-butene-1,4-diyl)bis- & $\mathrm{C} 16 \mathrm{H} 16$ & 208 & 20.5 \\
\hline 48 & 19.08 & 194 & 1,2-Diphenylcyclopropane & $\mathrm{C} 15 \mathrm{H} 14$ & 194 & 63.5 \\
\hline 49 & 19.30 & 91 & Benzene, 1,1'-(1,4-but anediyl)bis- & $\mathrm{C} 16 \mathrm{H} 18$ & 210 & 92.1 \\
\hline 50 & 19.38 & 119 & $\begin{array}{c}\text { Benzene, } \\
1,1^{\prime} \text {-(1,1,2,2-tetramethyl-1,2-ethanediyl)bis }\end{array}$ & $\mathrm{C} 18 \mathrm{H} 22$ & 238 & 33.5 \\
\hline 51 & 19.53 & 105 & Pentane, 1,4-diphenyl- & $\mathrm{C} 17 \mathrm{H} 20$ & 224 & 88.6 \\
\hline 52 & 19.74 & 115 & Benzene, 1,1'-(1-but enylidene)bis- & $\mathrm{C} 16 \mathrm{H} 16$ & 208 & 31.5 \\
\hline 53 & 20.02 & 117 & Benzene, 1,1'-(1-butene-1,4-diyl)bis-, (Z)- & $\mathrm{C} 16 \mathrm{H} 16$ & 208 & 35.4 \\
\hline 54 & 20.51 & 204 & 2-Phenylnaphthalene & $\mathrm{C} 16 \mathrm{H} 12$ & 204 & 28.1 \\
\hline 55 & 20.69 & 105 & Benz[a] anthracene, 7,12-dihydro- & $\mathrm{C} 18 \mathrm{H} 14$ & 230 & 23.1 \\
\hline 56 & 21.35 & 91 & 1,6-Heptadiene, 2-methyl-6-phenyl- & $\mathrm{C} 14 \mathrm{H} 18$ & 186 & 12.0 \\
\hline 57 & 21.41 & 118 & $\begin{array}{c}\text { Benzene, } \\
1,1^{\prime} \text {-(1,4-dimethyl-1-butene-1,4-diyl)bis- }\end{array}$ & $\mathrm{C} 18 \mathrm{H} 20$ & 236 & 34.8 \\
\hline 58 & 21.65 & 219 & 2,3-Diazabicyclo[2.2.1]hept-2-ene, & $\mathrm{C} 17 \mathrm{H} 16 \mathrm{~N} 2$ & 248 & 59.6 \\
\hline
\end{tabular}




\begin{tabular}{|c|c|c|c|c|c|c|}
\hline & & & & & & \\
\hline 59 & 21.71 & 204 & 2-Phenylnaphthalene & $\mathrm{C} 16 \mathrm{H} 12$ & 204 & 49.3 \\
\hline 60 & 21.83 & 218 & 9-Phenyl-5H-benzocycloheptene & $\mathrm{C} 17 \mathrm{H} 14$ & 218 & 24.8 \\
\hline 61 & 21.92 & 91 & $\begin{array}{l}\text { 1-Phenyl-4-(3,5-dimethylphenyl)buta-1,3- } \\
\text { diene }\end{array}$ & $\mathrm{C} 18 \mathrm{H} 18$ & 234 & 59.5 \\
\hline 62 & 22.57 & 217 & $\begin{array}{c}\text { 5-(2-Propenylidene)-1 } 0,11 \text {-dihydro-5H-dib } \\
\text { enzo[a,d]cycloheptene }\end{array}$ & $\mathrm{C} 18 \mathrm{H} 16$ & 232 & 58.2 \\
\hline 63 & 22.93 & 218 & 9-Phenyl-5H-benzocycloheptene & $\mathrm{C} 17 \mathrm{H} 14$ & 218 & 28.2 \\
\hline 64 & 23.34 & 230 & p-Terphenyl & $\mathrm{C} 18 \mathrm{H} 14$ & 230 & 68.0 \\
\hline 65 & 23.71 & 230 & p-Terphenyl & $\mathrm{C} 18 \mathrm{H} 14$ & 230 & 51.3 \\
\hline 66 & 24.17 & 244 & 1,1':4',1"-Terphenyl-, 3'-methyl- & $\mathrm{C} 19 \mathrm{H} 16$ & 244 & 65.8 \\
\hline 67 & 24.52 & 91 & $\begin{array}{l}\text { Benzenemethanamine, } \\
\mathrm{N} \text {-(phenylmethylene)- }\end{array}$ & $\mathrm{C} 14 \mathrm{H} 13 \mathrm{~N}$ & 195 & 25.2 \\
\hline 68 & 24.59 & 91 & $\begin{array}{c}\text { Benzene, } \\
1,1^{\prime} \text {-[3-(2-phenylethylidene)-1,5-pentanedi } \\
\text { yl]bis- }\end{array}$ & $\mathrm{C} 25 \mathrm{H} 26$ & 326 & 19.3 \\
\hline 69 & 25.61 & 91 & Benzene, 1,1'-(1,3-propanediyl)bis- & $\mathrm{C} 15 \mathrm{H} 16$ & 196 & 29.2 \\
\hline 70 & 25.77 & 91 & Benzene, 1,1'-(1,3-propanediyl)bis- & $\mathrm{C} 15 \mathrm{H} 16$ & 196 & 57.0 \\
\hline 71 & 26.60 & 91 & Benzene, 1,1'-(1,4-butanediyl)bis- & $\mathrm{C} 16 \mathrm{H} 18$ & 210 & 7.03 \\
\hline 72 & 27.82 & 306 & $1,1^{\prime}: 2^{\prime}, 1^{\prime \prime}: 2^{\prime \prime}, 1$ '"'-Quaterphenyl $^{\prime}$ & $\mathrm{C} 24 \mathrm{H} 18$ & 306 & 26.8 \\
\hline 73 & 28.35 & 91 & $\begin{array}{c}1,3,5,7,9,11,13,15,17,19,21,23 \text {-Cyclotetrac } \\
\text { osadodecaene }\end{array}$ & $\mathrm{C} 24 \mathrm{H} 24$ & 312 & 37.9 \\
\hline 74 & 29.70 & 306 & 1,1':3',1"-Terphenyl, 5'-phenyl- & $\mathrm{C} 24 \mathrm{H} 18$ & 306 & 26.6 \\
\hline
\end{tabular}

Table 2. FT-IR Spectrum of PS waste plast ic to heavy fuel oil funct ional group name

\begin{tabular}{cccccc}
\hline Number of Wave & $\begin{array}{c}\text { Wave Number } \\
(\mathrm{cm}-1)\end{array}$ & Functional Group & Number ofWave & $\begin{array}{c}\text { Wave Number } \\
(\mathrm{cm}-1)\end{array}$ & Functional Group \\
\hline 5 & 3608.40 & Free OH (Sharp) & 18 & 1705.73 & Non-Conjugated \\
6 & 3442.03 & Intermolecular H & 19 & 1689.24 & Non-Conjugated \\
8 & 2733.02 & Bonds (broad) & & 1602.89 & Non-Conjugated \\
16 & 2185.34 & C-CH3 & 20 & 1454.86 & CH2 \\
17 & 2163.67 & C-C=-C-C=-CH & 21 & 1378.12 & CH3 \\
19 & 1870.23 & Non-Conjugated & 23 & 1031.42 & Acetates \\
16 & 1803.12 & Non-Conjugated & 27 & 906.67 & $-\mathrm{CH}=\mathrm{CH} 2$ \\
17 & 1744.42 & Non-Conjugated & 28 & 695.23 & $-\mathrm{CH}=\mathrm{CH}-(\mathrm{cis})$ \\
\hline
\end{tabular}

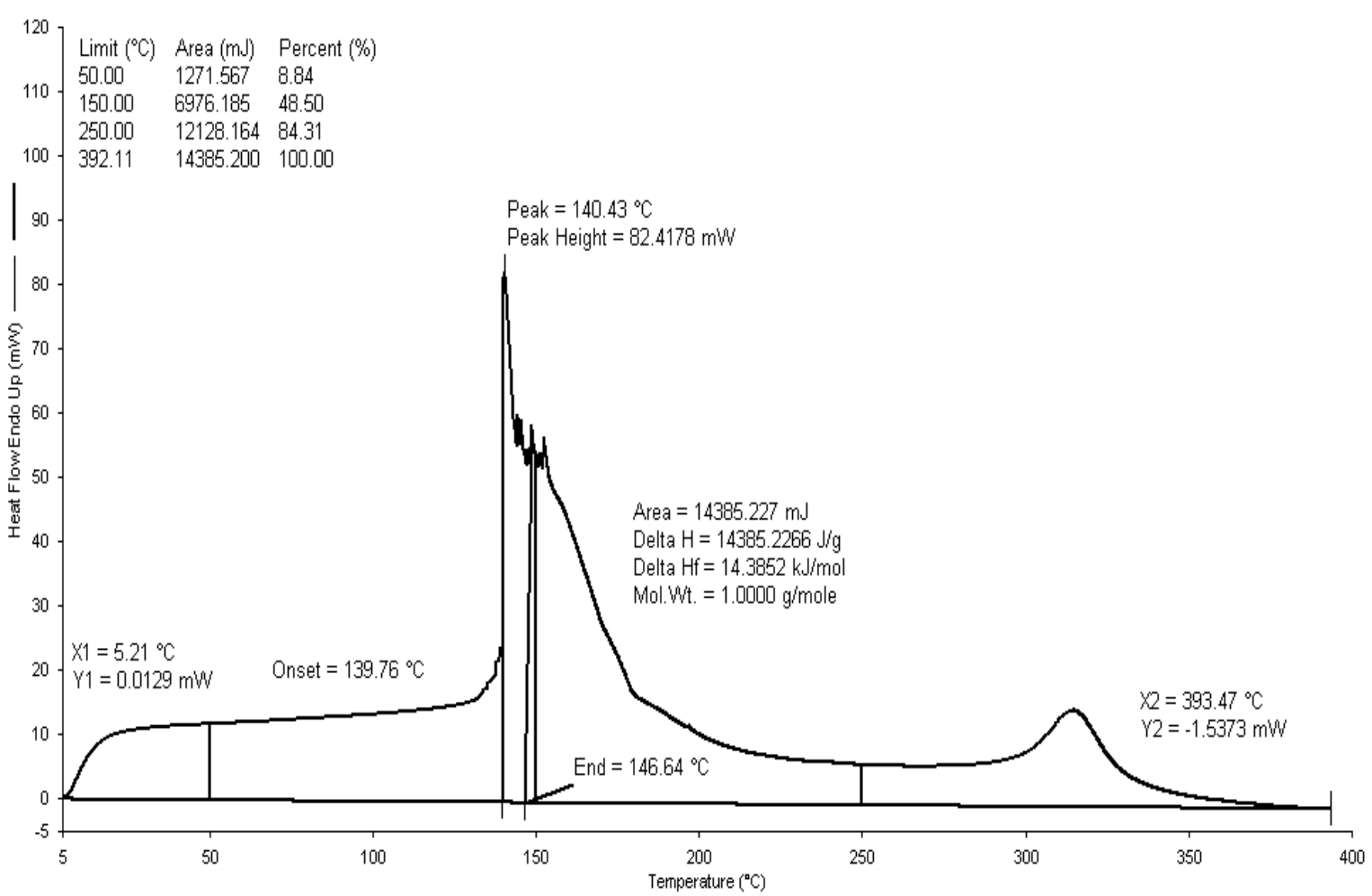

Figure 3. DSC graph of PS waste plastic to heavy fuel oil 


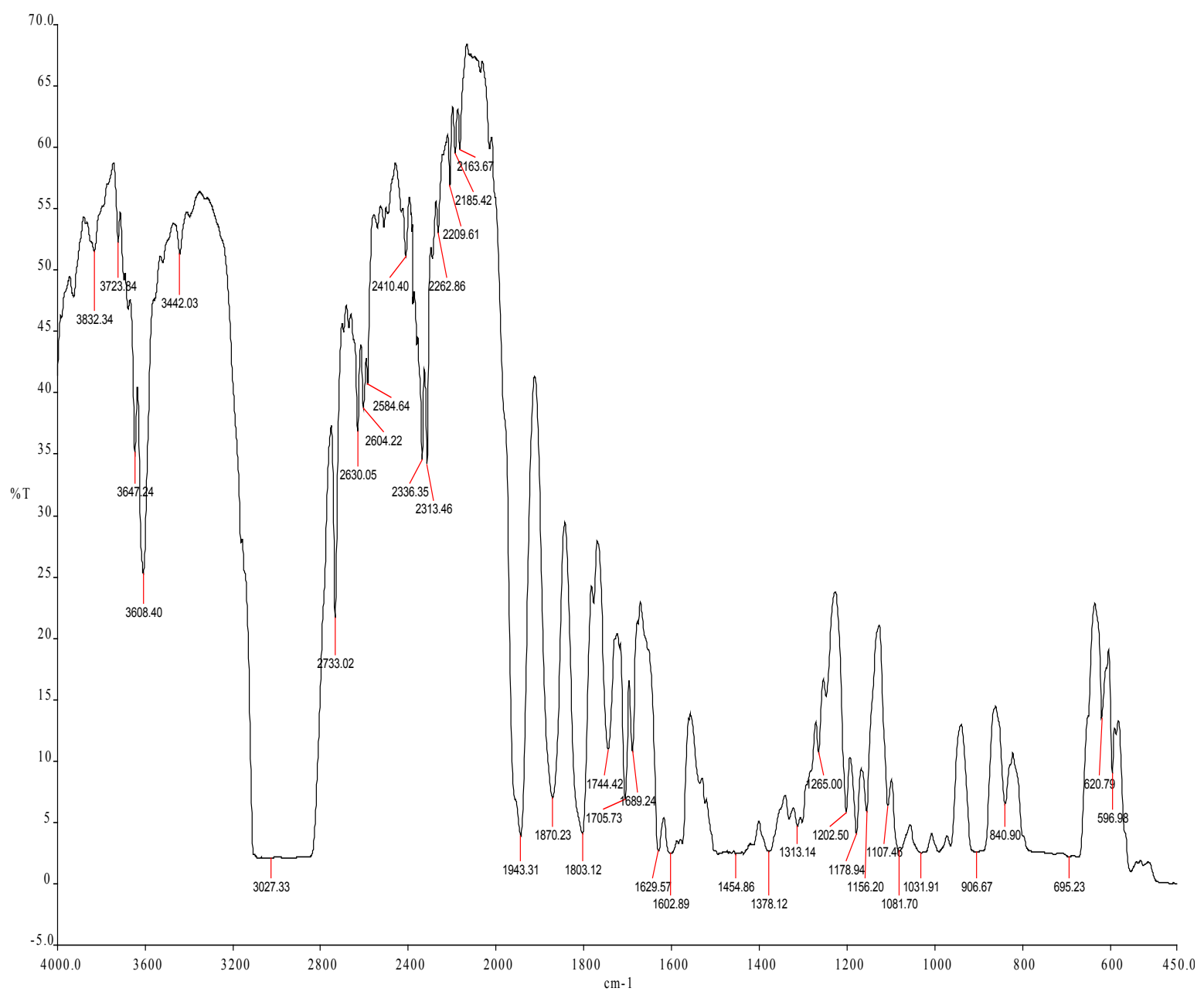

Figure 4. FT-IR Spectrum of PS waste plastic to heavy fuel oil

By using of Perkin Elmer FT-IR (spectrum 100) analysis of PS waste plastic to heavy hydrocarbon fuel (fig. 4 and table 2) according to their wave number and spectrum band following types of functional groups are appeared in the analysis. In the spectrum field we noticed that higher wave number are emerged in the in itial phase and middle index of the spectrum and in higher wave number small and bulky both functional groups are available and in low wave number double bond and single bond functional groups are available such as methane group, cis and trans alkene etc. Hereafter wave number $3608.40 \mathrm{~cm}^{-1}$, functional group is Free $\mathrm{OH}$ (Sharp), wave number $3442.03 \mathrm{~cm}^{-1}$ functional group is, Intermolecular $\mathrm{H}$ bonds (broad), wave nu mber $2733.02 \mathrm{~cm}^{-1}$ functional group is $\mathrm{C}_{-} \mathrm{CH}_{3}$, wave number 1870.81 $\mathrm{cm}^{-1}, 1802.23 \mathrm{~cm}^{-1}, 1745.41 .08 \mathrm{~cm}^{-1}, 1705.73 \mathrm{~cm}^{-1}, 1689.24$ $\mathrm{cm}^{-1}$ and $1602.89 \mathrm{~cm}^{-1}$ functional group is Non-Conjugated, wave number $1454.86 \mathrm{~cm}^{-1}$ and $1378.12 \mathrm{~cm}^{-1}$ functional group is $\mathrm{CH}_{3}$, wave number $1031.42 \mathrm{~cm}^{-1}$ functional group is Acetates, wave number $906.67 \mathrm{~cm}^{-1}$ functional group is $-\mathrm{CH}=\mathrm{CH}_{2}$ and ultimately wave number $695.23 \mathrm{~cm}^{-1}$ functional group is $-\mathrm{CH}=\mathrm{CH}$-(cis) etc. Energy values are calculated, using formula is $\mathrm{E}=\mathrm{h} v$, Where $\mathrm{h}=\mathrm{P}$ lanks Constant, $\mathrm{h}=6.626 \times 10^{-34} \mathrm{~J}, v=$ Frequency in Hert $\mathrm{z}\left(\mathrm{sec}^{-1}\right)$, Where $v=\mathrm{c} / \lambda$, $\mathrm{c}=$ Speed of light, where, $\mathrm{c}=3 \times 10^{10} \mathrm{~m} / \mathrm{s}, \mathrm{W}=1 / \lambda$, where $\lambda$ is wave length and $\mathrm{W}$ is wave number in $\mathrm{cm}^{-1}$. Therefore the equation $\mathrm{E}=\mathrm{h} v$, can substitute by the following equation, $\mathrm{E}=\mathrm{hcW}$. According to their wave number several energy values are calculated such as for $2733.02\left(\mathrm{~cm}^{-1}\right)$ calculated energy, $E=5.42 \times 10^{-20} \mathrm{~J}$. Similarly, wave number 1870.23 $\left(\mathrm{cm}^{-1}\right)$ energy, $\mathrm{E}=3.71 \times 10^{-20} \mathrm{~J}$, wave nu mber $1378.12\left(\mathrm{~cm}^{-1}\right)$ energy, $\mathrm{E}=2.73 \times 10^{-20} \mathrm{~J}$ and eventually wave number 906.67 $\left(\mathrm{cm}^{-1}\right)$ functional group is $1.80 \times 10^{-20} \mathrm{~J}$ respectively.

\section{Conclusions}

Polystyrene waste plastic to heavy fuel production by thermal degradation and fractional column process was used and temperature range was for fractional distillation colu mn $340-365^{\circ} \mathrm{C}$. Produced fuel was analysis by GC/MS and FT-IR. From GC/MS analysis result indicates that produced fuel has aromatic hydrocarbon chain range is $\mathrm{C}_{6}$ to $\mathrm{C}_{25}$. FT-IR analysis result showed band energy value which is represent as calorific value into fuel and DSC result showed heat enthalpy value. Produced fuel has lots of benzene group compound such as Benzene, Ethylbenzene, Styrene, $\alpha$-Methylstyrene and $p$-Terphenyl etc. This fuel has also combination of aliphatic and aromatic group. From the experiment and analysis results, it can be shown that a very 
good conversion at temperature range $200-450{ }^{\circ} \mathrm{C}$ for liquefaction of polystyrene waste and maximizing the liquid yield products. Thus, thermal liquefaction of post consumer polystyrene plastic waste at high temperature can be used as the first stage for converting post-consumer plastics wastes into clean heavy fuel oil. By using this technology can be solve polystyrene waste plastic problem and landfill problems in the environment and it could be boost up alternative energy sector as well as foreign fuel oil dependency.

\section{ACKNOWLWDGEMENTS}

The author acknowledges the support of Dr. Karin Kaufman, the founder and sole owner of Natural State Research, Inc. The authors also acknowledge the valuable contributions NSR laboratory team members during the preparation of this manuscript.

\section{REFERENCES}

[1] Williams, P. T.; Williams, E. A. J. Anal. Appl. Pyroly sis 1999, 51, 107-126.

[2] Ramdoss, P. K.; Tarrer, A. R. Fuel 1998, 77 (4), 293-299.

[3] McCaffrey, W. C.; Cooper, D. G.; Kamal, M. R. Polym. Degrad.Stab. 1998, 62, 513-521.

[4] Garforth, A. A.; Lin, Y.-H.; Sharratt, P. N.; Dwyer, J. App1. Catal.A: General 1998, 169, 331-342.
[5] Shabtai, J.; Xiao, X.; Zmierczak, W. Energy Fuels 1997, 11, 76-87.

[6] Sharratt, P. N.; Lin, Y.-H.; Garforth, A. A.; Dwyer, J. Ind. Eng.Chem. Res. 1997, 36, 5118-5124.

[7] Park, D. W.; Hwang, E. Y.; Kim, J. R.; Choi, J. K.; Kim, Y. A.; Woo, H. C. Polym. Degrad. Stab. 1999, 65, 193-198.

[8] Uemichi, Y.; Takuma, K.; Ayame, A. Chem. Commun. 1998, 1975-1976.

[9] [Buekens, A. G.; Huang, H. Resour. Conserv. Recycl. 1998, 23, 163-181.

[10] Selhan K.; Jale Y.; Suat U.; Chunshan S. Catalytic Coprocessing of Low-Density Poly ethy lene with VGO Using Metal Supported on Activated Carbon. Energy \& Fuels 2002, 16, 1301-1308.

[11] An Analysis of Plastics Consumption and Recovery in Europe, 2002-2003; Association of Plastics Manufactures in Europe (APME): Brussels, Belgium.

[12] Miskolczi, N.; Bartha, L.; Antal, F.; Dudas, Cs. Talanta 2005, $66,1264-1271$

[13] De la Puente, G.; Sedran, U. Appl. Catal., B 1998, 19, 305-311.

[14] Yanik, J.; Uddin, Md. A.; Sakata, Y. Energy Fuels 2001, 15, 163- 169.

[15] Speight, J. G. New approaches to hydroprocessing. Catal. Today.2004, 98 (1), 55-60.

[16] Rana, M. S.; Samano, V.; Ancheyta, J.; Diaz, J. A. I. A review of recent advances on process technologies for upgrading of heavy oils and residua. Fuel 2007, 86 (9), 1216-1231 